\title{
Delay-Dependent Stability Criterion of Caputo Fractional Neural Networks with Distributed Delay
}

\author{
Abdulaziz Alofi, ${ }^{1}$ Jinde Cao, ${ }^{1,2}$ Ahmed Elaiw, ${ }^{1}$ and Abdullah Al-Mazrooei ${ }^{1}$ \\ ${ }^{1}$ Department of Mathematics, Faculty of Science, King Abdulaziz University, Jeddah 21589, Saudi Arabia \\ ${ }^{2}$ Department of Mathematics, Southeast University, Nanjing 210096, China \\ Correspondence should be addressed to Jinde Cao; jdcao@seu.edu.cn
}

Received 18 November 2013; Accepted 26 November 2013; Published 12 January 2014

Academic Editor: Guanghui Wen

Copyright (C) 2014 Abdulaziz Alofi et al. This is an open access article distributed under the Creative Commons Attribution License, which permits unrestricted use, distribution, and reproduction in any medium, provided the original work is properly cited.

\begin{abstract}
This paper is concerned with the finite-time stability of Caputo fractional neural networks with distributed delay. The factors of such systems including Caputo's fractional derivative and distributed delay are taken into account synchronously. For the Caputo fractional neural network model, a finite-time stability criterion is established by using the theory of fractional calculus and generalized Gronwall-Bellman inequality approach. Both the proposed criterion and an illustrative example show that the stability performance of Caputo fractional distributed delay neural networks is dependent on the time delay and the order of Caputo's fractional derivative over a finite time.
\end{abstract}

\section{Introduction}

It is well known that the fractional calculus is a generalization and extension of the traditional integer-order differential and integral calculus. The fractional calculus has gained importance in both theoretical and engineering applications of several branches of science and technology. It draws a great application in nonlinear oscillations of earthquakes and many physical phenomena such as seepage flow in porous media and in fluid dynamic traffic models. Many practical systems in interdisciplinary fields can be described through fractional derivative formulation. For more details on fractional calculus theory, one can see the monographs of Miller and Ross [1], Podlubny [2], Diethelm [3], and Kilbas et al. [4]. In the last few years, there has been a surge in the study of the theory of fractional dynamical systems. Some recent works the theory of fractional differential systems can be seen in [5-10] and references therein. In particular, for the first time, Lazarević [7] investigated the finite-time stability of fractional time-delay systems. In [8], Lazarević and Spasić further introduced the Gronwall's approach to discuss the finite-time stability of fractional-order dynamic systems.

Compared with the classical integer-order derivatives, fractional-order derivatives provide an excellent approach for the description of memory and hereditary properties of various processes. Therefore, it may be more accurate to model by fractional-order derivatives than integer-order ones. In [11-13], fractional operators were introduced into artificial neural network, and the fractional-order formulations of artificial neural network models were also proposed in research works about biological neurons. Recently, there has been an increasing interest in the investigation of the fractional-order neural networks, and some important and interesting results were obtained [13-19], due to their significance in both theory and applications. In [13], Kaslik and Sivasundaram discussed the stability and multistability, periodic oscillation, bifurcations, and chaos of fractionalorder neural networks of Hopfield type. Arena et al. [14] investigated the chaotic behavior in noninteger-order cellular neural networks. Boroomand and Menhaj [15] considered fractional-order Hopfield neural networks and analyzed their stability by means of energy-like function. Huang et al. [16] presented the complex dynamical behaviors of a fractionalorder four-cell cellular neural network by means of numerical simulations.

On the other hand, time delay is one of the inevitable problems in practical engineering applications, which has an important effect on the stability and synchronization capability of dynamical systems in the real world. In recent years, 
there are many important results with respect to integerorder network dynamical systems (see [20-26] and references therein). In $[20,21]$, the finite-time synchronization problems of various kinds of integer-order dynamical system without delay effect have been investigated. Currently, the dynamical behaviours of integer-order networks dynamical systems with delay [22-26] are discussed by applying the different methods. However, to the best of our knowledge, there are very rare works on the problems for fractionalorder delayed neural networks [18, 19]. Zhou et al. [18] discussed numerical simulation of chaotic synchronization of a fractional neuron network system with time-varying delays, while the theoretical result was not established. Chen et al. [19] investigated the uniform stability for a class of fractionalorder neural networks with constant delay by the analytical approach.

In this paper, motivated by the works of Lazarević and Spasić [7, 8], we are devoted to establishing the finitetime stability criterion for fractional-order neural networks with distributed delay. Since fractional-order derivatives are nonlocal and have weakly singular kernels, many methods applied to the classical integer-order dynamical systems are not suitable for fractional-order delayed neural networks. Therefore, it is quite interesting and challenging to study the stability problems for fractional-order distributed delay neural networks. In this paper, we will apply the fractional calculus and generalized Gronwall-Bellman inequality (see [9]) to establish the finite-time stability criterion of fractional-order distributed delayed neural networks. The obtained criterion is convenient and feasible to check the considered model's stability over a finite time.

This paper is organized as follows. In Section 2, we will recall some definitions concerning fractional calculus and elaborate the Caputo fractional distributed delayed neural networks. In Section 3, the finite-time stability criterion of Caputo fractional neural networks with distributed delays is established. An example is given to show the effectiveness and applicability of the proposed result in Section 4. Finally, some concluding remarks are drawn in Section 5.

\section{Preliminaries and Model Formulation}

In this section, we first recall some definitions of fractional calculus and the well-known results. For more details, one can see [1-4]. Next, we elaborate Caputo fractional neural networks model with distributed delay.

Definition 1. Riemann-Liouville's fractional integral of order $q>0$ with the lower limit zero for a function $f: \mathbb{R}^{+} \rightarrow \mathbb{R}^{n}$ is defined as

$$
D^{-q} f(t)=\frac{1}{\Gamma(q)} \int_{0}^{t}(t-s)^{q-1} f(s) d s, \quad t>0,
$$

that provided the right side is pointwise defined on $[0,+\infty)$, where $\Gamma(\cdot)$ is the gamma function.
Definition 2. Caputo's fractional derivative of order $q$ for a function $f: \mathbb{R}^{+} \rightarrow \mathbb{R}^{n}$ is defined as

$$
\begin{aligned}
D^{q} f(t)= & \frac{1}{\Gamma(m-q+1)} \\
& \quad \times \int_{0}^{t}(t-s)^{m-q} f^{(m+1)}(s) d s \\
& \quad 0 \leq m \leq q<m+1 .
\end{aligned}
$$

Definition 3. The Mittag-Leffler function in two parameters is defined as

$$
E_{\alpha, \beta}(z)=\sum_{k=0}^{+\infty} \frac{z^{k}}{\Gamma(k \alpha+\beta)},
$$

where $\alpha>0, \beta>0$, and $z \in \mathbb{C} ; \mathbb{C}$ denotes the complex plane. In particular, for $\beta=1$,

$$
E_{\alpha, 1}(z)=E_{\alpha}(z)=\sum_{k=0}^{+\infty} \frac{z^{k}}{\Gamma(k \alpha+1)}
$$

have the interesting property

$$
D^{\alpha} E_{\alpha}\left(\lambda z^{\alpha}\right)=\lambda E_{\alpha}\left(\lambda z^{\alpha}\right), \quad \lambda, z \in \mathbb{C} .
$$

From [1-4], one knows that the superiority of Caputo's fractional derivative is that the initial conditions for fractional differential equations under Caputo's sense take on the similar form as for integer-order differential ones, which also have well understood physical meanings. Furthermore, Caputo's fractional derivative of a constant is equal to zero. Therefore, we discuss fractional-order neural networks with distributed delay under Caputo's sense throughout this paper.

In this paper, we are interested in the finite-time stability of Caputo's fractional-order neural networks with distributed delay by the following state equations:

$$
\begin{array}{r}
D^{\alpha} y_{i}(t)=-c_{i} y_{i}(t)+\sum_{j=1}^{n} a_{i j} \tilde{f}_{j}\left(y_{j}(t)\right) \\
+\sum_{j=1}^{n} \int_{0}^{\tau} b_{i j}(s) \tilde{g}_{j}\left(y_{j}(t-s)\right) d s+I_{i}, \\
i=1,2, \ldots, n, \quad t \geq 0,
\end{array}
$$

with the initial conditions

$$
y_{i}(\theta)=\widetilde{\varphi}_{i}(\theta), \quad i=1,2, \ldots, n, \theta \in[-\tau, 0],
$$

or in the matrix-vector notation

$$
\begin{aligned}
D^{\alpha} y(t)= & -C y(t)+A f(y(t)) \\
& +\int_{0}^{\tau} B(s) g(y(t-s)) d s+I, \quad t \geq 0,
\end{aligned}
$$

with the initial condition

$$
y(\theta)=\tilde{\varphi}(\theta), \quad \theta \in[-\tau, 0],
$$


where $D^{\alpha} x$ is an $\alpha$ order Caputo's fractional derivative of $x$; $\alpha$ is a positive constant and satisfies $0<\alpha<1$; $n$ is the number of neurons in the indicated neural network; $y(t)=$ $\left(y_{1}(t), y_{2}(t), \ldots, y_{n}(t)\right)^{T}$ is the state vector of the network at time $t$; the functions

$$
\begin{aligned}
& f(y(t))=\left(\tilde { f } _ { 1 } \left(y(t), \tilde{f}_{2}\left(y(t), \ldots, \tilde{f}_{n}(y(t))^{T},\right.\right.\right. \\
& g(y(t))=\left(\tilde { g } _ { 1 } \left(y(t), \tilde{g}_{2}\left(y(t), \ldots, \tilde{g}_{n}(y(t))^{T}\right.\right.\right.
\end{aligned}
$$

are the activation functions of the neurons at time $t ; C=$ $\operatorname{diag}\left(c_{1}, c_{2}, \ldots, c_{n}\right)$ is a diagonal matrix with $c_{i}>0$ for $i=$ $1,2, \ldots, n ; A=\left(a_{i j}\right)_{n \times n}$ is the feedback matrix; $\tau>0$ denotes the maximum possible transmission delay from neuron to another; $B(s)=\left(b_{i j}(s)\right)_{n \times n}$ is the delayed feedback matrix; $I=\left(I_{1}, I_{2}, \ldots, I_{n}\right)^{T}$ is an external bias vector.

Throughout this paper, we make the following assumptions.

$\left(H_{1}\right)$ The neurons activation functions $\tilde{f}_{j}(\cdot)$ and $\widetilde{g}_{j}(\cdot)$ are bounded.

$\left(H_{2}\right)$ The neurons activation functions $\widetilde{f}_{j}(\cdot)$ and $\widetilde{g}_{j}(\cdot)$ are Lipschitz continuous. That is, there exist positive constants $M_{j}, N_{j}(j=1,2, \ldots, n)$ such that

$$
\begin{array}{r}
\left|\tilde{f}_{j}(u)-\tilde{f}_{j}(v)\right| \leq M_{j}|u-v|, \\
\left|\tilde{g}_{j}(u)-\tilde{g}_{j}(v)\right| \leq N_{j}|u-v|, \\
\forall u, v \in \mathbb{R} .
\end{array}
$$

$\left(H_{3}\right)$ For $i, j=1,2, \ldots, n$, the function $b_{i j}(\cdot)$ is continuous on $[0, \tau]$.

From the assumptions above, we denote $M=$ $\max _{1 \leq j \leq n}\left\{M_{j}\right\}, N=\max _{1 \leq j \leq n}\left\{N_{j}\right\}$, and $B=\sup _{0 \leq s \leq \tau}\{\|B(s)\|\}$.

Note that Caputo's fractional derivative of a constant is equal to zero [2], and then it follows from Schauder fixed point theorem and assumptions $\left(H_{1}\right)-\left(H_{3}\right)$ that the equilibrium points of system (1) exist. We can shift the equilibrium point of system (6) to the origin. Let $y^{*}=$ $\left(y_{1}^{*}, y_{2}^{*}, \ldots, y_{n}^{*}\right)^{T}$ be an equilibrium point of system (6); $x(t)=\left(x_{1}(t), x_{2}(t), \ldots, x_{n}(t)\right)^{T}=\left(y_{1}(t)-y_{1}^{*}, y_{2}(t)-\right.$ $\left.y_{2}^{*}, \ldots, y_{n}(t)-y_{n}^{*}\right)^{T}$; then system (1) can be written as

$$
\begin{array}{r}
D^{\alpha} x_{i}(t)=-c_{i} x_{i}(t)+\sum_{j=1}^{n} a_{i j} f_{j}\left(x_{j}(t)\right) \\
+\sum_{j=1}^{n} \int_{0}^{\tau} b_{i j}(s) g_{j}\left(x_{j}(t-s)\right) d s, \\
\quad i=1,2, \ldots, n, \quad t \geq 0,
\end{array}
$$

with the initial conditions

$$
x_{i}(\theta)=\varphi_{i}(\theta), \quad i=1,2, \ldots, n, \quad \theta \in[-\tau, 0],
$$

where $f_{j}\left(x_{j}(t)\right)=\tilde{f}_{j}\left(x_{j}(t)+y_{j}^{*}\right)-\tilde{f}_{j}\left(y_{j}^{*}\right), g_{j}\left(x_{j}(t)\right)=$ $\tilde{g}_{j}\left(x_{j}(t)+y_{j}^{*}\right)-\tilde{g}_{j}\left(y_{j}^{*}\right)$, and $\varphi_{i}(\theta)=y_{i}(\theta)-y_{i}^{*}, \theta \in[-\tau, 0]$.

By using the matrix-vector notation, system (12) can be expressed in the form

$$
\begin{aligned}
D^{\alpha} x(t)= & -C x(t)+A F(x(t)) \\
& +\int_{0}^{\tau} B(s) G(x(t-s)) d s, \quad t \geq 0,
\end{aligned}
$$

with the initial condition

$$
x(\theta)=\varphi(\theta), \quad \theta \in[-\tau, 0],
$$

where $F(x(t))=\left(f_{1}\left(x_{1}(t)\right), f_{2}\left(x_{2}(t)\right), \ldots, f_{n}\left(x_{n}(t)\right)^{T}\right.$, and $G(x(t))=\left(g_{1}\left(x_{1}(t)\right), g_{2}\left(x_{2}(t)\right), \ldots, g_{n}\left(x_{n}(t)\right)^{T}\right.$.

From assumption $\left(\mathrm{H}_{2}\right)$, we know that the functions $f_{i}$ and $g_{i}$ satisfy the following properties:

$$
\begin{array}{r}
\left|f_{i}(u)\right| \leq M_{i}|u|, \quad\left|g_{i}(u)\right| \leq N_{i}|u|, \\
i=1,2, \ldots, n, \forall u \in \mathbb{R} .
\end{array}
$$

Define the two new functions as follows:

$$
\begin{aligned}
& r_{i}(t)= \begin{cases}\frac{f_{i}\left(x_{i}(t)\right)}{x_{i}(t)}, & x_{i}(t) \neq 0, \\
0, & x_{i}(t)=0,\end{cases} \\
& k_{i}(t)= \begin{cases}\frac{g_{i}\left(x_{i}(t)\right)}{x_{i}(t)}, & x_{i}(t) \neq 0, \\
0, & x_{i}(t)=0 .\end{cases}
\end{aligned}
$$

It follows from (17) that

$$
f_{i}\left(x_{i}(t)\right)=r_{i}(t) x_{i}(t), \quad g_{i}\left(x_{i}(t)\right)=k_{i}(t) x_{i}(t) .
$$

Thus, system (14) can be further shifted into the following form:

$$
\begin{array}{r}
D^{\alpha} x(t)=[-C+A R(t)] x(t) \\
+\int_{0}^{\tau} B(s) K(t-s) x(t-s) d s, \\
t \geq 0,0<\alpha<1,
\end{array}
$$

where $R(t)=\operatorname{diag}\left\{r_{i}(t)\right\}$, and $K(t)=\operatorname{diag}\left\{k_{i}(t)\right\}$. Obviously, one can get $\left|r_{i}(t)\right| \leq M_{i}$, and $\left|k_{i}(t)\right| \leq N_{i}$.

Let $\mathbf{C}\left([-\tau, 0], \mathbb{R}^{n}\right)$ denote the Banach space of all continuous functions over a time interval of length $\tau$, mapping the interval $[t-\tau, t]$ into $\mathbb{R}^{n}$ with the norm defined as follows: for $\operatorname{every} \varphi(\cdot) \in \mathbf{C}\left([-\tau, 0], \mathbb{R}^{n}\right)$,

$$
\|\varphi\|=\sup _{\theta \in[-\tau, 0]}|\varphi(\theta)| .
$$

Definition 4 (see [7, 8], finite-time stability). System (19) with the initial condition $x(t)=\varphi(t),-\tau \leq t \leq 0$ is finite-time stable with respect to $\left\{\delta, \varepsilon, t_{0}, J\right\}, \delta<\varepsilon$, if and only if

$$
\|\varphi\|<\delta
$$


implies

$$
\|x(t)\|<\varepsilon, \quad \forall t \in J
$$

where $\delta$ is a positive real number and $\varepsilon>0, \delta<\varepsilon, t_{0}$ denotes the initial time of observation of the system, and $J$ denotes time interval $J=\left[t_{0}, t_{0}+H\right)$.

The following generalized Gronwall-Bellman inequality was derived by Ye et al. [9], which is basic to establish the finite-time stability criterion of system (19).

Lemma 5 (see [9, generalized Gronwall-Bellman inequality]). Let $u(t), a(t)$ be nonnegative and local integrable on $[0, H), H \leq+\infty$, and let $g(t)$ be a nonnegative, nondecreasing continuous function defined on $[0, H), g(t) \leq M$, and let $M$ be a real constant, $\alpha>0$ with

$$
u(t) \leq a(t)+g(t) \int_{0}^{t}(t-s)^{\alpha-1} u(s) d s, \quad t \in[0, H)
$$

and then

$$
\begin{array}{r}
u(t) \leq a(t) \\
+\int_{0}^{t}\left\{\sum_{n=1}^{+\infty} \frac{[g(t) \Gamma(\alpha)]^{n}}{\Gamma(n \alpha)}(t-s)^{n \alpha-1} a(s)\right\} d s, \\
t \in[0, H) .
\end{array}
$$

In addition, if $a(t)$ is a nondecreasing function $[0, T)$, then

$$
u(t) \leq a(t) E_{\alpha}\left[g(t) \Gamma(\alpha) t^{\alpha}\right], \quad t \in[0, H),
$$

where $E_{\alpha}(\cdot)$ is the Mittag-Leffler function with one parameter (see [1-4]).

\section{Main Result}

In this section, we derive the sufficient conditions for finitetime stability of Caputo fractional neural networks with distributed delays by using the generalized Gronwall-Bellman inequality [9].

Theorem 6. Let system (19) satisfy Assumptions $\left(H_{1}\right)-\left(H_{3}\right)$ with the initial condition $x(\theta)=\varphi(\theta), \theta \in[-\tau, 0]$, and

$$
\begin{aligned}
& {\left[1+\frac{\tau N B}{\Gamma(\alpha+1)} t^{\alpha}\right]} \\
& \quad \times E_{\alpha}\left[(\mu(C)+M \mu(A)+\tau N B) t^{\alpha}\right] \leq \frac{\varepsilon}{\delta}, \quad t \in J=[0, H),
\end{aligned}
$$

and then system (19) is finite-time stable with respect to $\{\delta, \varepsilon, 0, J\}, \delta<\varepsilon$, where $\mu(\cdot)$ denotes the largest singular value of matrix $(\cdot)$ and $E_{\alpha}(\cdot)$ is the Mittag-Leffler function with one parameter.
Proof. According to the property of the fractional order $0<$ $\alpha<1$, one can obtain that system (19) is equivalent to the following Volterra fractional integral with memory

$$
\begin{aligned}
x(t)= & \varphi(0) \\
& +\frac{1}{\Gamma(\alpha)} \int_{0}^{t}(t-s)^{\alpha-1} \\
& \times\{[-C+A R(s)] x(s) \\
& \left.\quad+\int_{0}^{\tau} B(\theta) K(s-\theta) x(s-\theta) d \theta\right\} d s .
\end{aligned}
$$

Applying the appropriate properties of the norm $\|\cdot\|$ on (27), it follows that

$$
\begin{aligned}
\|x(t)\| \leq\|\varphi\| & +\frac{1}{\Gamma(\alpha)} \\
& \times \int_{0}^{t}(t-s)^{\alpha-1} \\
& \times \|[-C+A R(s)] x(s) \\
& \quad+\int_{0}^{\tau} B(\theta) K(s-\theta) x(s-\theta) d \theta \| d s .
\end{aligned}
$$

Let $u(t)=\sup _{\theta \in[-\tau, 0]}\|x(t+\theta)\|$. For $0 \leq t<H$, it follows from (28) that

$$
\begin{aligned}
& u(t) \leq\|\varphi\|+\frac{1}{\Gamma(\alpha)} \\
& \times \int_{0}^{t}(t-s)^{\alpha-1}\{[\mu(C)+M \mu(A)] \cdot\|x(s)\| \\
& \left.+\int_{0}^{\tau} B N\|x(s-\theta)\| d \theta\right\} d s \\
& \leq\|\varphi\|+\frac{1}{\Gamma(\alpha)} \\
& \times \int_{0}^{t}(t-s)^{\alpha-1}\{[\mu(C)+M \mu(A)] \cdot\|x(s)\| \\
& \left.+\int_{0}^{\tau} B N[u(s)+\|\varphi\|] d \theta\right\} d s \\
& \leq\|\varphi\|+\frac{1}{\Gamma(\alpha)} \\
& \times \int_{0}^{t}(t-s)^{\alpha-1}\{[\mu(C)+M \mu(A)] \cdot u(s) \\
& \left.+\int_{0}^{\tau} B N[u(s)+\|\varphi\|] d \theta\right\} d s
\end{aligned}
$$




$$
\begin{aligned}
\leq & \|\varphi\|\left[1+\frac{\tau B N}{\Gamma(\alpha+1)} t^{\alpha}\right]+\frac{1}{\Gamma(\alpha)} \\
& \times \int_{0}^{t}(t-s)^{\alpha-1}[\mu(C)+M \mu(A)+\tau B N] u(s) d s,
\end{aligned}
$$

where $\mu(\cdot)$ denotes the largest singular value of matrix $(\cdot)$. Obviously, one can introduce a nondecreasing function $a(t)$ as

$$
a(t)=\|\varphi\|\left[1+\frac{\tau B N}{\Gamma(\alpha+1)} t^{\alpha}\right], \quad t \in[0, H) .
$$

An application of the lemma yields that

$$
\begin{aligned}
\|x(t)\| \leq & u(t) \\
\leq & \|\varphi\|\left[1+\frac{\tau B N}{\Gamma(\alpha+1)} t^{\alpha}\right] \\
& \quad \times E_{\alpha}\left[(\mu(C)+M \mu(A)+\tau B N) t^{\alpha}\right], \quad t \in[0, H) .
\end{aligned}
$$

Hence, using the basic condition of the theorem, inequality (26) yields

$$
\|x(t)\|<\varepsilon, \quad \forall t \in J .
$$

This completes the proof.

Remark 7. The obtained theorem presents a finite-time stability criterion, which shows that the finite-time stability of Caputo fractional distributed delayed neural networks is dependent on the time delay and the order of Caputo's fractional derivative.

\section{An Illustrative Example}

In this section, we give an example to verify the validity and applicability of the given result.

Example 1. Consider the following two-state Caputo fractional neural networks model with distributed delay:

$$
\begin{aligned}
D^{\alpha} x_{1}(t)= & -0.4 x_{1}(t)-0.2 f_{1}\left(x_{1}(t)\right)+0.1 f_{2}\left(x_{2}(t)\right) \\
& -\int_{0}^{\tau} s f_{1}\left(x_{1}(t-s)\right) d s+\int_{0}^{\tau} s^{2} f_{2}\left(x_{2}(t-s)\right) d s, \\
D^{\alpha} x_{2}(t)= & -0.3 x_{2}(t)+0.3 f_{1}\left(x_{1}(t)\right)+0.2 f_{2}\left(x_{2}(t)\right) \\
& +\int_{0}^{\tau} s^{2} f_{1}\left(x_{1}(t-s)\right) d s+\int_{0}^{\tau} s f_{2}\left(x_{2}(t-s)\right) d s,
\end{aligned}
$$

with an associated function of the initial state

$$
x(t)=\varphi(t)=0, \quad-\tau \leq t \leq 0,
$$

where $\alpha=1 / 2, \tau=0.2, f_{j}\left(x_{j}\right)=g_{j}\left(x_{j}\right)=(1 / 2)\left(\left|x_{j}+1\right|-\right.$ $\left.\left|x_{j}-1\right|\right)$, and $j=1,2$.
Now, we apply our theorem to verify that system (33) is finite-time stable. Take

$$
\begin{gathered}
t_{0}=0, \quad J=[0,2), \quad \delta=0.02, \quad \varepsilon=0.1, \\
C=\left[\begin{array}{cc}
-0.4 & 0 \\
0 & -0.3
\end{array}\right], \quad A=\left[\begin{array}{cc}
-0.2 & 0.1 \\
0.3 & 0.2
\end{array}\right], \\
B(s)=\left[\begin{array}{cc}
-s & s^{2} \\
s^{2} & s
\end{array}\right] .
\end{gathered}
$$

Then, it follows from the initial data and system (33) that

$$
\begin{gathered}
M=N=1, \quad \mu(C)=0.5, \\
\mu(A)=1.2108, \quad B=0.2040 .
\end{gathered}
$$

Using the condition of theorem, we can obtain

$$
\begin{gathered}
{\left[1+\frac{0.0408}{0.886} T_{e}^{0.5}\right] E_{1 / 2}\left[1.7516 T_{e}^{0.5}\right]} \\
\leq \frac{0.1}{0.02} \Longrightarrow T_{e} \approx 1.0361
\end{gathered}
$$

where $T_{e}$ is an estimated time of the finite-time stability. Therefore, system (33) is finite-time stable with respect to $\{0.02,0.1,0,[0,2)\}$.

\section{Conclusions}

In this paper, we have investigated the finite-time stability of Caputo fractional distributed delayed neural networks and have derived the finite-time stability criterion based on the fractional calculus theory and generalized GronwallBellman inequality technique. The proposed criterion with an illustrative example shows that the stability performance of Caputo fractional neural networks with distributed delay is dependent on the time delay and the order of Caputo's fractional derivative over a finite time. Also, some other dynamical behaviors, such as synchronization and control, of fractional-order network systems will become our future investigative works.

\section{Conflict of Interests}

The authors declare that there is no conflict of interests regarding the publication of this paper.

\section{Acknowledgments}

This work was funded by the Deanship of Scientific Research (DSR), King Abdulaziz University (KAU), under Grant 3130/1434/HiCi. Therefore, the authors acknowledge technical and financial support of KAU.

\section{References}

[1] K. S. Miller and B. Ross, An Introduction to the Fractional Calculus and Fractional Differential Equations, John Wiley \& Sons, New York, NY, USA, 1993. 
[2] I. Podlubny, Fractional Differential Equations, vol. 198 of Mathematics in Science and Engineering, Technical University of Kosice, Kosice, Slovak Republic, 1999.

[3] K. Diethelm, The Analysis of Fractional Differential Equations, vol. 2004, Springer, Berlin, Germany, 2010.

[4] A. A. Kilbas, H. M. Srivastava, and J. J. Trujillo, Theory and Applications of Fractional Differential Equations, vol. 204 of NorthHolland Mathematics Studies, Elsevier Science B.V., Amsterdam, The Netherlands, 2006.

[5] V. Lakshmikantham, "Theory of fractional functional differential equations," Nonlinear Analysis. Theory, Methods \& Applications, vol. 69, no. 10, pp. 3337-3343, 2008.

[6] V. Lakshmikantham and A. S. Vatsala, "Basic theory of fractional differential equations," Nonlinear Analysis. Theory, Methods \& Applications, vol. 69, no. 8, pp. 2677-2682, 2008.

[7] M. P. Lazarević, "Finite time stability analysis of $P D^{\alpha}$ fractional control of robotic time-delay systems," Mechanics Research Communications, vol. 33, no. 2, pp. 269-279, 2006.

[8] M. P. Lazarević and A. M. Spasić, "Finite-time stability analysis of fractional order time-delay systems: Gronwall's approach," Mathematical and Computer Modelling, vol. 49, no. 3-4, pp. 475481, 2009.

[9] H. Ye, J. Gao, and Y. Ding, "A generalized Gronwall inequality and its application to a fractional differential equation," Journal of Mathematical Analysis and Applications, vol. 328, no. 2, pp. 1075-1081, 2007.

[10] H. Zhang, J. Cao, and W. Jiang, "General solution of linear fractional neutral differential difference equations," Discrete Dynamics in Nature and Society, vol. 2013, Article ID 489521, 7 pages, 2013.

[11] B. N. Lundstrom, M. H. Higgs, W. J. Spain, and A. L. Fairhall, "Fractional differentiation by neocortical pyramidal neurons," Nature Neuroscience, vol. 11, no. 11, pp. 1335-1342, 2008.

[12] G. A. Anastassiou, "Fractional neural network approximation," Computers \& Mathematics with Applications, vol. 64, no. 6, pp. 1655-1676, 2012.

[13] E. Kaslik and S. Sivasundaram, "Nonlinear dynamics and chaos in fractional-order neural networks," Neural Networks, vol. 32, pp. 245-256, 2012.

[14] P. Arena, L. Fortuna, and D. Porto, "Chaotic behavior in noninteger-order cellular neural networks," Physical Review E, vol. 61, no. 1, pp. 776-781, 2000.

[15] A. Boroomand and M. B. Menhaj, "Fractional-order Hopfield neural networks," Lecture Notes in Computer Science, vol. 5506, no. 1, pp. 883-890, 2009.

[16] X. Huang, Z. Zhao, Z. Wang, and Y. Li, "Chaos and hyperchaos in fractional-order cellular neural networks," Neurocomputing, vol. 94, pp. 13-21, 2012.

[17] S. Zhou, H. Li, and Z. Zhu, "Chaos control and synchronization in a fractional neuron network system," Chaos, Solitons and Fractals, vol. 36, no. 4, pp. 973-984, 2008.

[18] S. Zhou, P. Hu, and H. Li, "Chaotic synchronization of a fractional neuron network system with time-varying delays," in Proceedings of the International Conference on Communications, Circuits and Systems (ICCCAS '09), pp. 863-867, July 2009.

[19] L. Chen, Y. Chai, R. Wu, T. Ma, and H. Zhai, "Dynamic analysis of a class of fractional-order neural networks with delay," Neurocomputing, vol. 111, pp. 190-194, 2013.

[20] X. Yang and J. Cao, "Finite-time stochastic synchronization of complex networks," Applied Mathematical Modelling, vol. 34, no. 11, pp. 3631-3641, 2010.
[21] N. Cai, W. Li, and Y. Jing, "Finite-time generalized synchronization of chaotic systems with different order," Nonlinear Dynamics, vol. 64, no. 4, pp. 385-393, 2011.

[22] M. Xiao, W. X. Zheng, and J. Cao, "Bifurcation and control in a neural network with small and large delays," Neural Networks, vol. 44, pp. 132-142, 2013.

[23] Z. Wang, J. Cao, G. Chen, and X. Liu, "Synchronization in an array of nonidentical neural networks with leakage delays and impulsive coupling," Neurocomputing, vol. 111, pp. 177-183, 2013.

[24] X. Yang, J. Cao, and J. Lu, "Synchronization of Markovian coupled neural networks with nonidentical node-delays and random coupling strengths," IEEE Transactions on Neural Networks and Learning Systems, vol. 23, no. 1, pp. 60-71, 2012.

[25] X. Yang and J. Cao, "Synchronization of discontinuous neural networks with delays via adaptive control," Discrete Dynamics in Nature and Society, vol. 2013, Article ID 147164, 9 pages, 2013.

[26] Y. Wang and J. Cao, "Cluster synchronization in nonlinearly coupled delayed networks of non-identical dynamic systems," Nonlinear Analysis. Real World Applications, vol. 14, no. 1, pp. 842-851, 2013. 


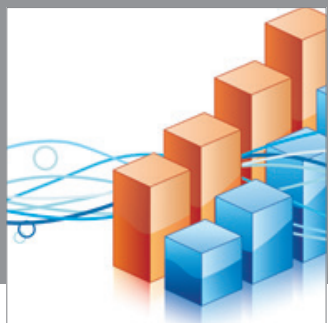

Advances in

Operations Research

mansans

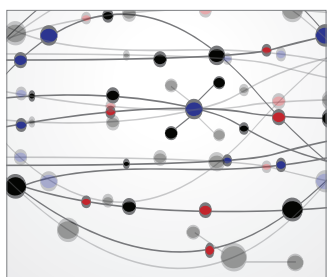

The Scientific World Journal
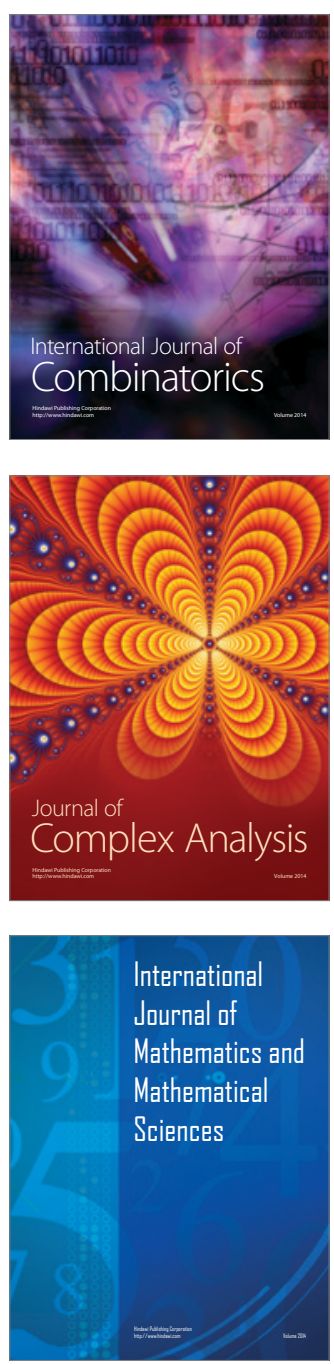
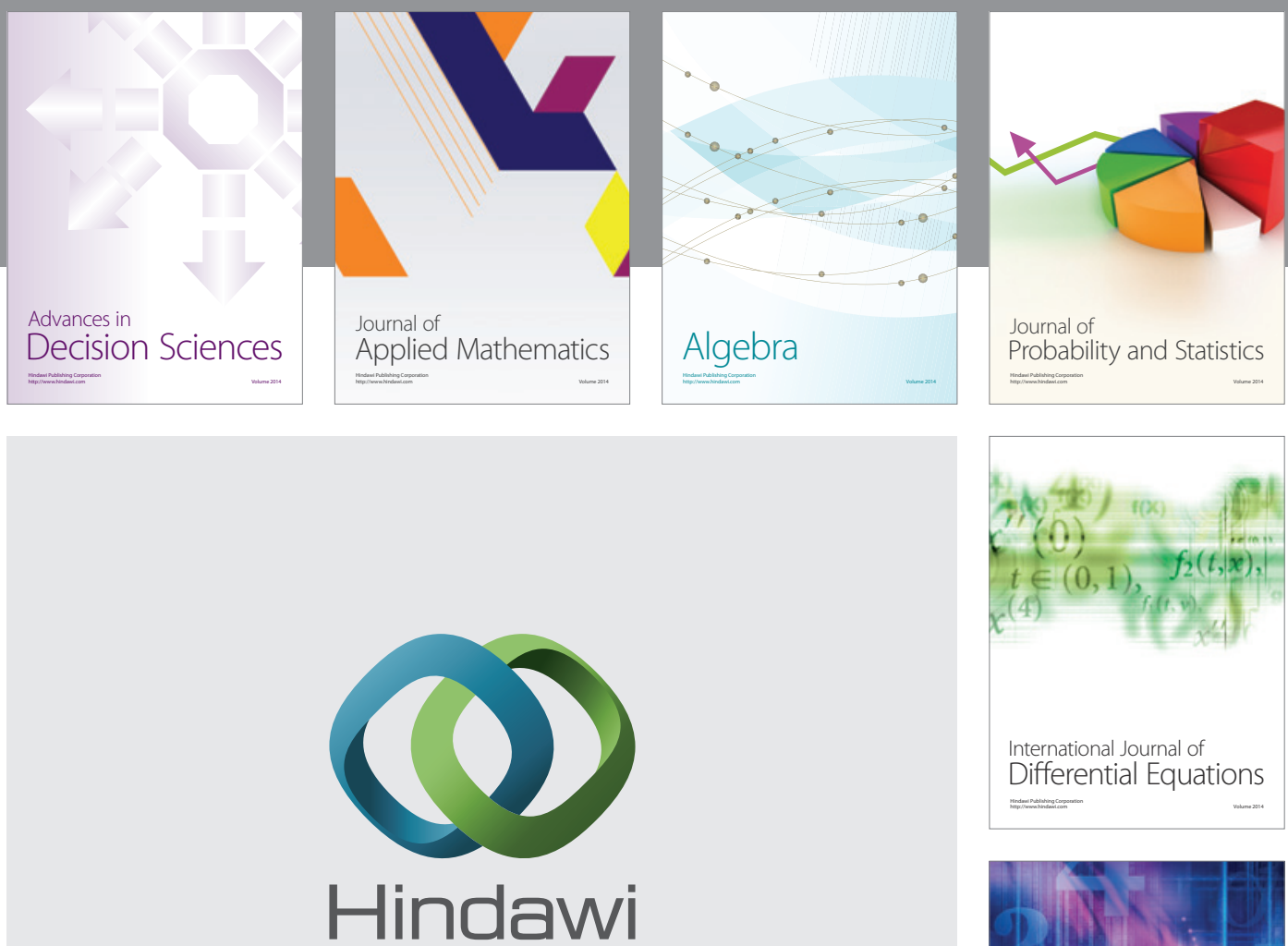

Submit your manuscripts at http://www.hindawi.com
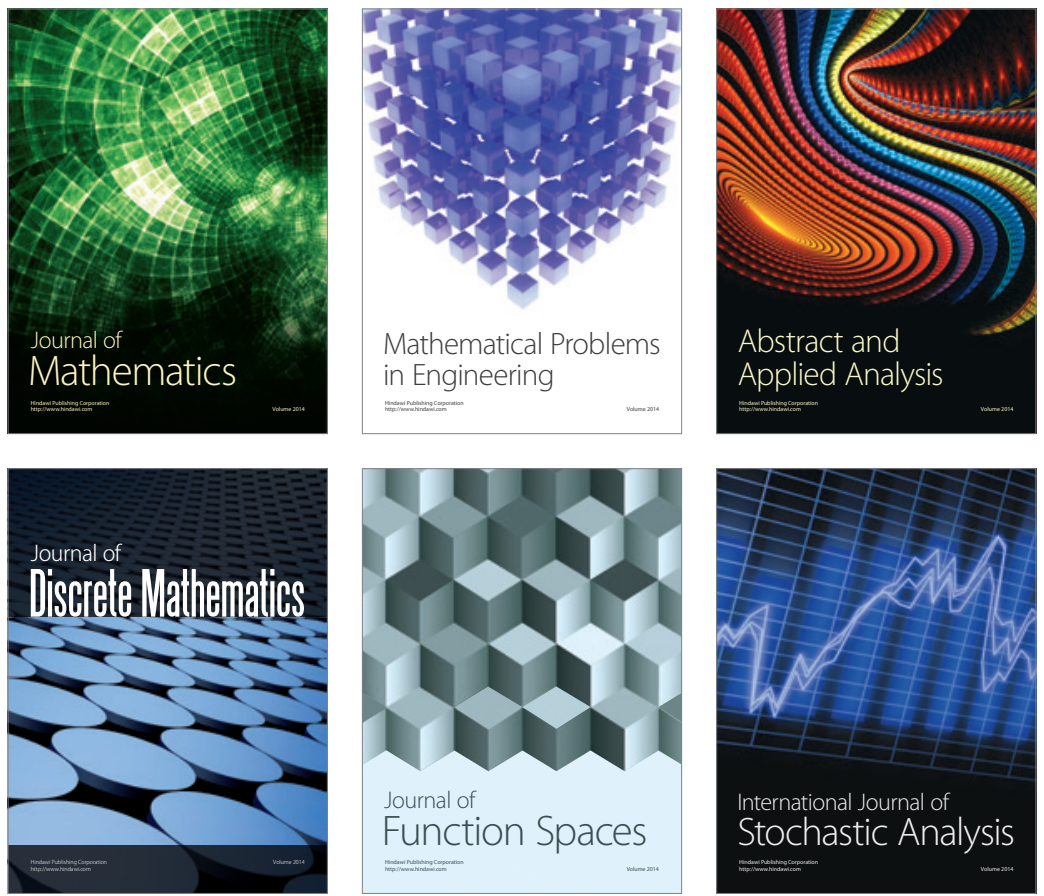

Journal of

Function Spaces

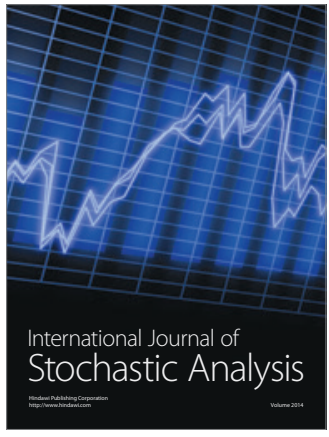

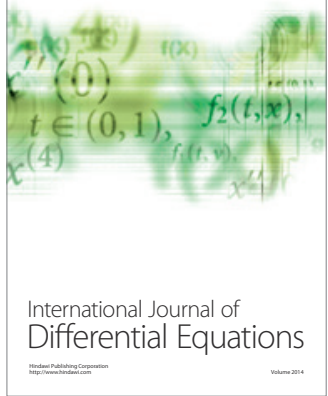
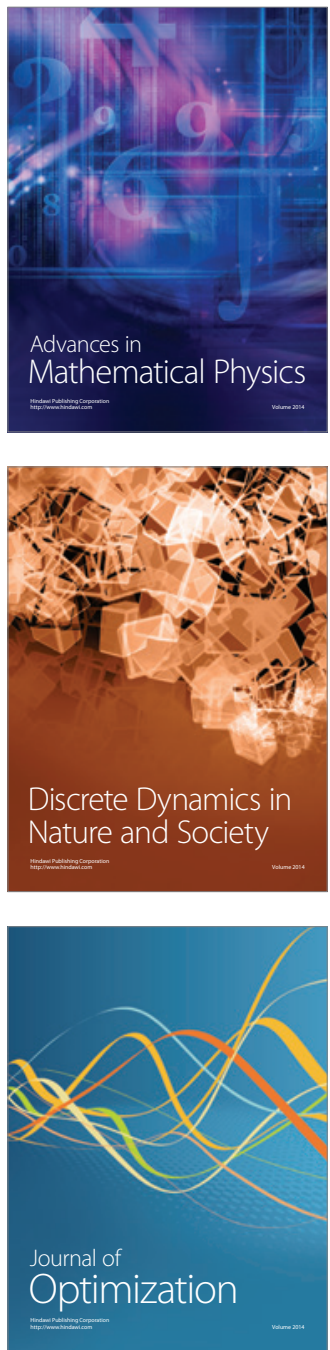